\title{
Global dynamics of a delayed chemostat model with harvest by impulsive flocculant input
}

\author{
Tongqian Zhang ${ }^{1,2,3}$, Wanbiao Ma ${ }^{{ }^{*}}$ and Xinzhu Meng ${ }^{2,3}$
}

\section{"Correspondence:}

Wanbiao_Ma@ustb.edu.cn

'School of Mathematics and

Physics, University of Science and

Technology Beijing, 30 Xue Yuan

Road, Beijing, 100083, China

Full list of author information is

available at the end of the article

\begin{abstract}
A mathematical model describing continuous microbial culture and harvest in a chemostat, incorporating a control strategy and defined by impulsive differential equations, is presented and investigated. Theoretical results indicate that the model has a microbe-extinction periodic solution, which is globally attractive if the threshold $R_{1}$ is less than unity, and the model is permanent if the threshold $R_{2}$ is greater than unity. Further, we consider the control strategy under time delay and periodical impulsive effect. Analysis shows that continuous microbial culture and harvest process can be implemented by adjusting time delay, impulsive period or input amount of flocculant. Finally, we give an example with numerical simulations to illustrate the control strategy.
\end{abstract}

MSC: 34A37; 34K45; 92B05

Keywords: chemostat model; microbial flocculation; time delay; impulsive effect; global attractivity; permanence; control strategy

\section{Introduction and model formulation}

A chemostat is a classical bioreactor for microbes culture and has been widely applied in the field of microbiology and bioengineering $[1,2]$. The chemostat model has attracted the attention of many scholars since it was introduced by Monod in 1942 [3]. These models include mathematical models [4-16] and experimental models [17-19]. A simple chemostat can be designed by using a pump or an overflow system (see Figure 1), by which the volume of the chemostat can be controlled either [20].

It was found that some microbes can produce flocculation under the action of flocculating agents. This phenomenon makes it possible to harvest microbes by flocculating agents [21-32]. Recently, based on a classical simple chemostat model in which a microbial species consumes a single growth limiting substrate [3, 8], Tai et al. [33] have proposed a delayed differential equations (DDEs) model to describe the process of microbial continuous culture and harvest as follows:

$$
\left\{\begin{array}{l}
\frac{d S(t)}{d t}=D\left(S_{0}-S(t)\right)-h_{1} x(t) S(t), \\
\frac{d x(t)}{d t}=h x(t-\tau) S(t-\tau)-D x(t)-h_{2} x(t) F(t), \\
\frac{d F(t)}{d t}=D\left(F_{0}-F(t)\right)-h_{3} x(t) F(t),
\end{array}\right.
$$

(C) The Author(s) 2017. This article is distributed under the terms of the Creative Commons Attribution 4.0 International License (http://creativecommons.org/licenses/by/4.0/), which permits unrestricted use, distribution, and reproduction in any medium, provided you give appropriate credit to the original author(s) and the source, provide a link to the Creative Commons license, and indicate if changes were made. 
(a)
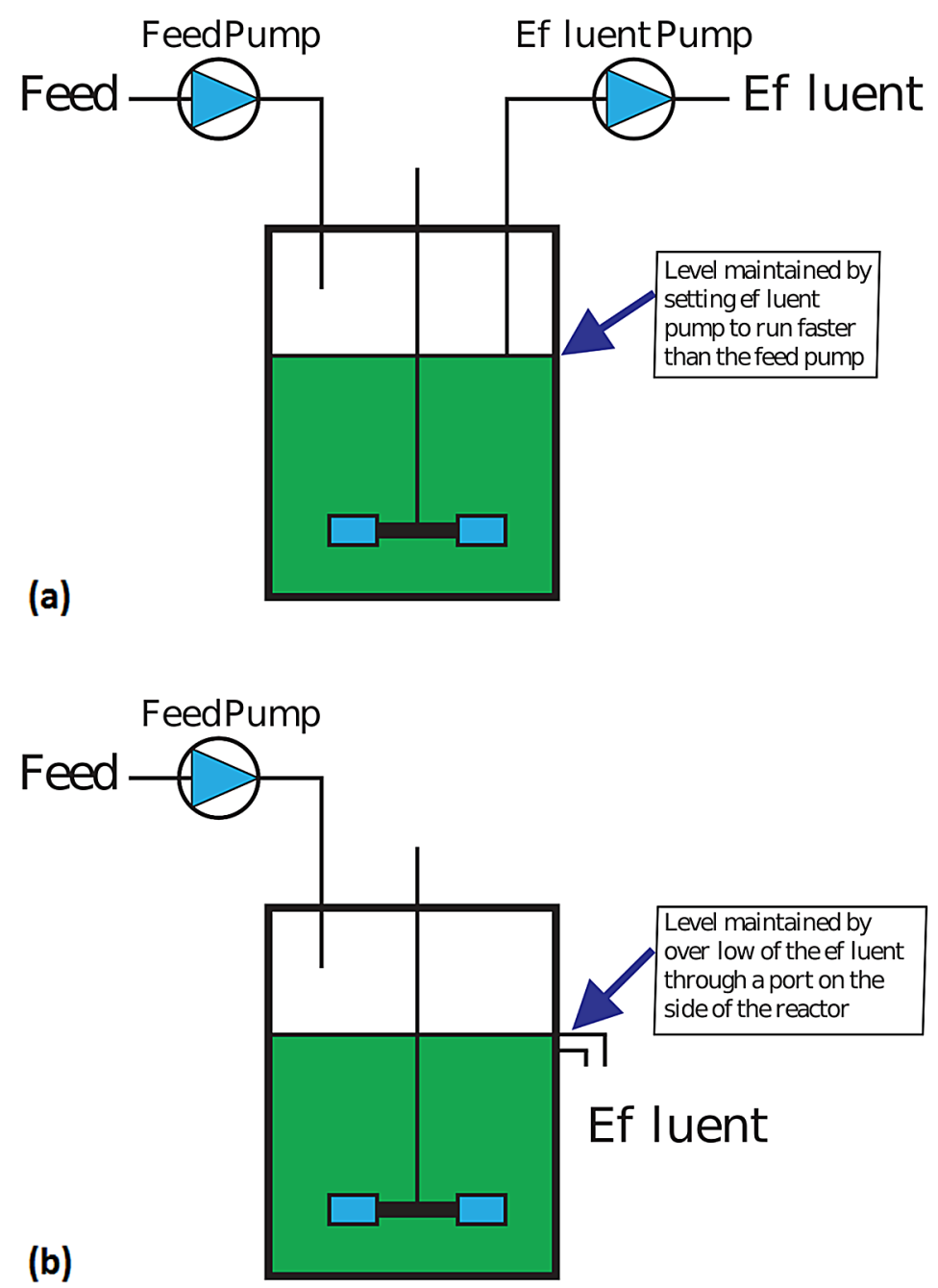

Figure 1 Two types of chemostat. (a) Type I chemostat. (b) Type II chemostat.

where $S(t), x(t)$ and $F(t)$ represent the concentration of the substrate, microbia and flocculating agent at time $t$, respectively. $D$ represents the velocity of medium, $S_{0}$ and $F_{0}$ represent the input concentration of substrate and flocculating agent, respectively. $h_{1}$ and $h$ represent the consumption of medium and the yield of microbia, respectively. $h_{3}$ is the loss rate of flocculant. $\tau$ represents the time involved converting nutrient into the microbia [34-45]. The authors found that the model can produce backward bifurcation and complex dynamics. By establishing analytic thresholds for the existence of backward bifurcation, they analyzed the local stability of the equilibria.

In model (1), the flocculating agent is assumed to be added into the chemostat continuously. While in practice, by considering resource savings and the growth cycle of microorganisms, the flocculating agent can be periodically added into the chemostat at some fixed moment. Thus we perfect the chemostat system by adding input channel of flocculants and output channel of flocculation by two pumps, respectively (see Figure 2). It can be regulated through inputting flocculant to flocculate microbia according to the concentration of microbia. This process can be described by impulsive differential equations (IDEs). Impulsive differential equations, on the one hand, can fully reflect the actual control situ- 


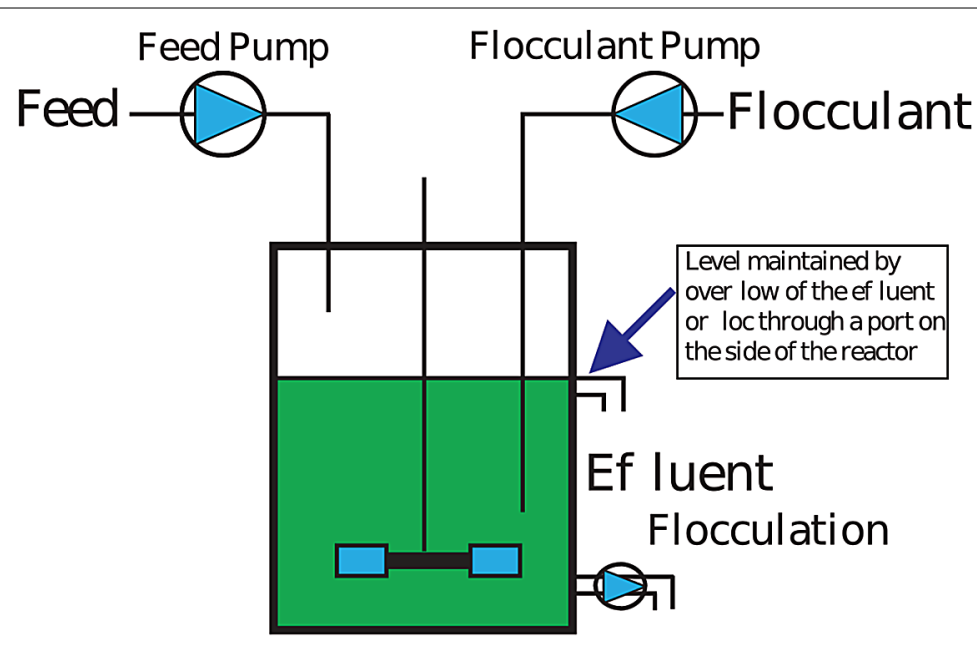

Figure 2 The modified chemostat.

ation; on the other hand, they can guide the operator to implement the impulsive control strategy conveniently and accurately [46-51]. Thus, we propose a new continuous culture chemostat model with time delay and impulsive harvest as follows:

$$
\left\{\begin{array}{l}
\frac{d S(t)}{d t}=D\left(S_{0}-S(t)\right)-h_{1} x(t) S(t), \\
\frac{d x(t)}{d t}=e^{-D \tau} h x(t-\tau) S(t-\tau)-D x(t)-h_{2} x(t) F(t), \\
\frac{d F(t)}{d t}=-D F(t)-h_{3} x(t) F(t), \\
S\left(t^{+}\right)=S(t), \\
x\left(t^{+}\right)=x(t), \\
F\left(t^{+}\right)=F(t)+\gamma F_{0},
\end{array}\right\} t=n T,
$$

where $T$ is the period of the impulsive effect, $\gamma F_{0}$ is the input amount of flocculant at every impulsive period $T . F\left(n T^{+}\right)=\lim _{t \rightarrow n T^{+}} F(t)$, and $F(t)$ is left continuous at $t=n T$, i.e., $F(n T)=\lim _{t \rightarrow n T^{-}} F(t), S(t), x(t)$ are continuous for all $t \geq 0$, the details can be seen in $[52,53]$.

Let $C_{+}=C\left([-\tau, 0], R_{+}^{3}\right)$ be the Banach space, $\psi=\left(\psi_{1}(s), \psi_{2}(s), \psi_{3}(s)\right)^{T}, \psi_{i}(\theta) \geq 0(-\tau \leq$ $\theta \leq 0, i=1,2,3)$ the initial conditions are given as

$$
\begin{aligned}
& S(\theta)=\psi_{1}(\theta), \quad x(\theta)=\psi_{2}(\theta), \quad F(\theta)=\psi_{3}(\theta), \\
& \psi \in C_{+}, \quad \psi_{i}(0)>0 \quad(i=1,2,3) .
\end{aligned}
$$

The rest of the paper is organized as follows. In Section 2, we briefly introduce some concepts and fundamental results, which are necessary for future discussion. In Section 3, we focus our attention on the global property of system (2), including the existence, global attractivity of the microbe-extinction periodic solution and the permanence of system (2). In Section 4, we give the threshold of key parameters of system (2) and discuss the control strategy. We finally give a conclusion and numerical simulations in Section 5, from which it can be seen that all simulations agree with the theoretical results. 


\section{Preliminaries}

In this section, we give some useful lemmas.

Let $f=\left(f_{1}, f_{2}, f_{3}\right)^{T}$ be the map defined by the right-hand side of the anterior three equations of system (2). Let $R_{+}=[0, \infty), R_{+}^{3}=\left\{x \in R^{3}: x \geq 0\right\}, \Omega=\operatorname{int} R_{+}^{3}$. Let $U: R_{+} \times R_{+}^{3} \rightarrow R_{+}$. If $U$ satisfies the following conditions: (1) $V$ is continuous in $((n-1) T, n T] \times R_{+}^{3}, n \in N$, and for each $x \in R_{+}^{3}, \lim _{(t, z) \rightarrow\left((n-1) T^{+}, x\right)} U(t, z)=U((n-1) T, x)$ and $\lim _{(t, z) \rightarrow\left(n T^{+}, x\right)} U(t, z)=$ $U\left(n T^{+}, x\right)$ exists; (2) $U$ is locally Lipschitzian in $x$. Then $U$ is said to belong to class $U_{0}$.

Lemma 2.1 ([52,53]) Let $U: R_{+} \times R_{+}^{3} \rightarrow R_{+}, H: R_{+} \times R_{+} \rightarrow R$ and $U \in U_{0}$. Assume that

$$
\begin{cases}D^{+} U(t, w(t)) \leq(\geq) H(t, U(t, w(t))), & t \neq n \omega, \\ U\left(t, w(t)^{+}\right) \leq(\geq) \Upsilon_{n}(U(t, w(t))), & t=n \omega,\end{cases}
$$

here $H$ is continuous in $(n \omega,(n+1) \omega] \times R_{+}$and $\forall x \in R_{+}, n \in N, \lim _{(t, y) \rightarrow\left((n \omega)^{+}, x\right)} H(t, y)=$ $H\left((n \omega)^{+}, x\right)$ exist $; \Upsilon_{n}: R_{+} \rightarrow R_{+}$is nondecreasing. Let $r(t)=r\left(t, 0, u_{0}\right)$ be the maximal (minimal) solution of the scalar impulsive differential equation

$$
\begin{cases}u^{\prime}=H(t, u), & t \neq n \omega, \\ u\left(t^{+}\right)=\Upsilon_{n}(u(t)), & t=n \omega,\end{cases}
$$

existing on $[0, \infty)$. Then $U\left(0^{+}, w_{0}\right) \leq(\geq) u_{0}$ implies that $U(t, w(t)) \leq(\geq) r(t), t \geq 0$, where $\omega(t)=\omega\left(t, 0, w_{0}\right)$ is any solution of $(4)$ existing on $[0, \infty)$.

Lemma 2.2 ([54]) Let $q_{1}, q_{2}, \tau$ be all positive constants and $z(t)>0$ for $t \in[-\tau, 0]$. Consider the following delay differential equation:

$$
\frac{d z(t)}{d t}=q_{1} z(t-\tau)-q_{2} z(t)
$$

then

(i) if $q_{1}<q_{2}$, then $\lim _{t \rightarrow \infty} z(t)=0$;

(ii) if $q_{1}>q_{2}$, then $\lim _{t \rightarrow \infty} z(t)=\infty$.

Lemma 2.3 ([52, 53]) Consider the following impulse differential inequalities:

$$
\begin{cases}u^{\prime}(t) \leq(\geq) a(t) u(t)+c(t), & t \neq t_{k}, \\ u\left(t_{k}^{+}\right) \leq(\geq) b_{k} u\left(t_{k}\right)+d_{k}, & t=t_{k}, k \in N\end{cases}
$$

where $a(t), c(t) \in C\left(R_{+}, R\right), b_{k} \geq 0$, and $d_{k}$ are constants. Assume

$\left(A_{0}\right)$ the sequence $\left\{t_{k}\right\}$ satisfies $0 \leq t_{0}<t_{1}<t_{2}<\cdots$, with $\lim _{t \rightarrow \infty} t_{k}=\infty$;

$\left(A_{1}\right) \quad u \in P C^{\prime}\left(R_{+}, R\right)$ and $u(t)$ is left-continuous at $t_{k}, k \in N$. Then

$$
\begin{gathered}
u(t) \leq(\geq) \\
u\left(t_{0}\right) \prod_{t_{0}<t_{k}<t} d_{k} \exp \left(\int_{t_{0}}^{t} a(s) d s\right)+\sum_{t_{0}<t_{k}<t}\left(\prod_{t_{k}<t_{j}<t} d_{j} \exp \left(\int_{t_{k}}^{t} a(s) d s\right)\right) d_{k} \\
+\int_{t_{0}}^{t} \prod_{s<t_{k}<t} b_{k} \exp \left(\int_{s}^{t} a(\theta) d \theta\right) c(s) d s, \quad t \geq t_{0} .
\end{gathered}
$$


Lemma 2.4 ([43]) Consider the following impulsive differential system:

$$
\begin{cases}\frac{d \hbar(t)}{d t}=r_{1}-r_{2} \hbar(t), & t \neq n T, \\ \hbar\left(t^{+}\right)=\hbar(t)+\mu, & t=n T,\end{cases}
$$

for each solution $\hbar(t)$ of $(6), \hbar(t) \rightarrow \hbar^{*}(t)$ as $t \rightarrow \infty$, where $\hbar^{*}(t)=\frac{r_{1}}{r_{2}}+\frac{\mu e^{-r_{2}(t-n T)}}{1-e^{-r_{2} T}}$ for $t \in$ $(n T,(n+1) T]$.

Lemma 2.5 There exist constants $M_{1}, M_{2}, M_{3}>0$ such that $S(t) \leq M_{1}, x(t) \leq M_{2}, F(t) \leq$ $M_{3}$ for each solution of (2) with all t large enough.

Proof Firstly, from the third and sixth equation of system (2), we have

$$
\begin{cases}\frac{d F(t)}{d t} \leq-F S(t), & t \neq n T, \\ F\left(t^{+}\right)=F(t)+\gamma F_{0}, & t=n T .\end{cases}
$$

By Lemma 2.3, we have $F(t) \leq \gamma F_{0} \frac{e^{D T}}{e^{D T}-1}+\varepsilon_{2}$ for $t$ large enough.

Let $V(t)=e^{-D \tau} \frac{h}{h_{1}} S(t-\tau)+x(t)$. It is clear that $V \in U_{0}$. Calculating the upper right derivative of $V(t)$ along a solution of system (2), one can get

$$
\frac{d V(t)}{d t} \leq e^{-D \tau} \frac{h D S_{0}}{h_{1}}-D V(t)
$$

then by Lemma 2.3, we have $\lim \sup _{t \rightarrow \infty} V(t) \leq e^{-D \tau} \frac{h S_{0}}{h_{1}}$, so $V(t)$ is ultimately bounded. Thus, $S(t)$ and $x(t)$ are ultimately bounded and $\lim \sup _{t \rightarrow \infty} S(t) \leq S_{0}, \limsup _{t \rightarrow \infty} x(t) \leq$ $e^{-D \tau} \frac{h S_{0}}{h_{1}}$. Let $M_{1}=S_{0}+\varepsilon, M_{2}=e^{-D \tau} \frac{h S_{0}}{h_{1}}+\varepsilon, M_{3}=\gamma F_{0} \frac{e^{D T}}{e^{D T}-1}+\varepsilon$, we have $S(t) \leq M_{1}, x(t) \leq M_{2}$, $F(t) \leq M_{3}$ for $t$ large enough. The proof is completed.

\section{Global dynamical analysis for system (2)}

In this section, we discuss the global dynamics of model (2), including the existence and global attractivity of the microbe-extinction periodic solution and the permanence.

\subsection{Existence and global attractivity of the microbe-extinction periodic solution}

Microbe-extinction solution describes that microbes are eventually absent from system (2), thus we let $x(t)=0$ in system (2), then system (2) changes to the following system:

$$
\left\{\begin{array}{l}
\frac{d S(t)}{d t}=D\left(S_{0}-S(t)\right), \\
\frac{d F(t)}{d t}=-D F(t), \\
S\left(t^{+}\right)=S(t), \\
F\left(t^{+}\right)=F(t)+\gamma F_{0},
\end{array}\right\} t \neq n T,
$$

Note that the variates $S(t)$ and $F(t)$ are independent of each other in system (7). Thus, by Lemma 2.4, we obtain that system (7) has a unique positive $T$-periodic solution 
$\left(S^{*}(t), F^{*}(t)\right)$ and for each solution $(S(t), F(t))$ of system (7), $S(t) \rightarrow S^{*}(t)$ and $F(t) \rightarrow F^{*}(t)$ as $t \rightarrow \infty$, where

$$
\left\{\begin{array}{l}
S^{*}(t)=S_{0} \\
F^{*}(t)=\frac{\gamma F_{0} e^{-D(t-n T)}}{1-e^{-D T}}
\end{array}\right.
$$

Therefore, we have the existence theorem for system (2).

Theorem 3.1 System (2) has a microbe-extinction periodic solution $\left(S_{0}, 0, F^{*}(t)\right)$.

Denote

$$
R_{1}=\frac{h e^{-D \tau} S_{0}}{D+h_{2} \frac{\gamma F_{0} e^{-\left(D+h_{3} M_{2}\right) T}}{1-e^{-\left(D+h_{3} M_{2}\right) T}} .}
$$

We have the following theorem about the attractivity of the microbe-extinction periodic solution of system (2).

Theorem 3.2 If $R_{1}<1$, then the microbe-extinction periodic solution $\left(S_{0}, 0, F^{*}(t)\right)$ of system (2) is globally attractive.

Proof Let $(S(t), x(t), F(t))$ be any solution of system (2) satisfying initial condition (3). Since $R_{1}<1$, one can choose $\varepsilon_{1}, \varepsilon_{2}>0$ such that

$$
h e^{-D \tau}\left(S_{0}+\varepsilon_{1}\right)<D+h_{2}\left(\frac{\gamma F_{0} e^{-\left(D+h_{3} M_{2}\right) T}}{1-e^{-\left(D+h_{3} M_{2}\right) T}}-\varepsilon_{2}\right) .
$$

By the first equation of system (2), we have

$$
\frac{d S(t)}{d t} \leq D\left(S_{0}-S(t)\right)
$$

According to Lemma 2.3, we have

$$
\limsup _{t \rightarrow \infty} S(t) \leq S_{0}
$$

Hence, there exists $n_{1} \in N^{+}$such that

$$
S(t) \leq S_{0}+\varepsilon_{1}
$$

for all $t \geq n_{1} T$, where $\varepsilon_{1}$ is an arbitrarily small positive constant.

By the third and sixth equations of system (2), we have

$$
\begin{cases}\frac{d F(t)}{d t} \geq-\left(D+h_{3} M_{2}\right) F(t), & t \neq n T, \\ F\left(t^{+}\right)=F(t)+\gamma F_{0}, & t=n T\end{cases}
$$


then consider the following impulsive differential system:

$$
\begin{cases}\frac{d q(t)}{d t}=-\left(D+h_{3} M_{2}\right) q(t), & t \neq n T, \\ q\left(t^{+}\right)=q(t)+\gamma F_{0}, & t=n T \\ q\left(0^{+}\right)=F\left(0^{+}\right) & \end{cases}
$$

Then, by using Lemma 2.1, we have $F(t) \geq q(t)$ and $q(t) \rightarrow q^{*}(t)$ as $t \rightarrow \infty, q^{*}(t)$ is the periodic solution of (12), where $q^{*}(t)=\frac{\gamma F_{0} e^{-\left(D+h_{3} M_{2}\right)(t-n T)}}{1-e^{-\left(D+h_{3} M_{2}\right) T}}, n T<t \leq(n+1) T$. By Lemma 2.4, we have that $q^{*}(t)$ is globally asymptotically stable. Hence there exists $n_{2} \in N^{+}$such that

$$
F(t) \geq q(t)>q^{*}(t)-\varepsilon_{2}>\frac{\gamma F_{0} e^{-\left(D+h_{3} M_{2}\right) T}}{1-e^{-\left(D+h_{3} M_{2}\right) T}}-\varepsilon_{2}
$$

for all $t \geq n_{2} T$, where $\varepsilon_{2}$ is an arbitrarily small positive constant.

From the second equation, (10) and (13), there exists a positive integer $n_{3}>\max \left\{n_{1}, n_{2}\right\}$, for $t>n_{3} T+\tau$, we have

$$
\frac{d x(t)}{d t} \leq h e^{-D \tau}\left(S_{0}+\varepsilon_{1}\right) x(t-\tau)-\left(D+h_{2} \frac{\gamma F_{0} e^{-\left(D+h_{3} M_{2}\right) T}}{1-e^{-\left(D+h_{3} M_{2}\right) T}}-\varepsilon_{2}\right) x(t) .
$$

Consider the following delay differential equation:

$$
\frac{d y(t)}{d t}=h e^{-D \tau}\left(S_{0}+\varepsilon_{1}\right) y(t-\tau)-\left(D+h_{2}\left(\frac{\gamma F_{0} e^{-\left(D+h_{3} M_{2}\right) T}}{1-e^{-\left(D+h_{3} M_{2}\right) T}}-\varepsilon_{2}\right)\right) y(t) .
$$

Since (9) holds, by Lemma 2.2, we get $\lim _{t \rightarrow \infty} y(t)=0$. Notice that for all $\theta \in[-\tau, 0]$, $x(\theta)=y(\theta)=\psi_{2}(\theta)>0$ holds. By the comparison theorem in differential equation and the positivity of solution (with $x(t) \geq 0$ ), we obtain $\lim _{t \rightarrow \infty} x(t)=0$.

Next, we will prove $\lim _{t \rightarrow \infty} S(t)=S_{0}$ and $\lim _{t \rightarrow \infty} F(t)=F^{*}(t)$. We assume that $0<x(t)<\varepsilon$ holds for all $t \geq 0$ in the following discussion without loss of generality. One the one hand, by the first equation of system (2), one gets

$$
\frac{d S(t)}{d t} \geq D S_{0}-\left(D+\varepsilon h_{1}\right) S(t)
$$

Then, we have $\liminf _{t \rightarrow \infty} S(t) \geq S_{0} \frac{D}{D+\varepsilon h_{1}}$. Thus, there exists $T>0$ such that for any $\varepsilon_{1}>0$,

$$
S(t) \geq S_{0} \frac{D}{D+\varepsilon h_{1}}-\varepsilon_{1}
$$

for $t>T$. Let $\varepsilon \rightarrow 0$, from (10) and (14) we have

$$
S_{0}-\varepsilon_{1}<S(t)<S_{0}+\varepsilon_{1}
$$

for $t$ large enough, then we have $\lim _{t \rightarrow \infty} S(t)=S_{0}$.

On the other hand, from the third and sixth equations of system (2), we have

$$
\begin{cases}\frac{d F(t)}{d t} \geq-\left(D+\varepsilon h_{3}\right) F(t), & t \neq n T, \\ F\left(t^{+}\right)=F(t)+\gamma F_{0}, & t=n T .\end{cases}
$$


Then we get the following comparison system:

$$
\begin{cases}\frac{d w_{1}(t)}{d t}=-\left(D+\varepsilon h_{3}\right) w_{1}(t), & t \neq n T \\ w_{1}\left(t^{+}\right)=w_{1}(t)+\gamma F_{0}, & t=n T \\ w_{1}\left(0^{+}\right)=F\left(0^{+}\right) & \end{cases}
$$

By Lemma 2.4, system (15) has a globally asymptotically stable positive periodic solution $w_{1}^{*}(t)$, where $w_{1}^{*}(t)=\frac{\gamma F_{0} e^{-\left(D+\varepsilon h_{3}\right)(t-n T)}}{1-e^{-\left(D+\varepsilon h_{3}\right) T}}$. Thus, by Lemma 2.1, we have $F(t) \geq w_{1}(t)$ and $w_{1}(t) \rightarrow$ $w_{1}^{*}(t)$ as $t \rightarrow \infty$. Therefore, there exists $T^{\prime}>0$ such that for any $\varepsilon_{2}>0$,

$$
F(t) \geq w_{1}^{*}(t)-\varepsilon_{2}
$$

for $t>T^{\prime}$. From the third and sixth equations of (2), one gets

$$
\begin{cases}\frac{d F(t)}{d t} \leq-D F(t), & t \neq n T, \\ F\left(t^{+}\right)=F(t)+\gamma F_{0}, & t=n T .\end{cases}
$$

Consider the following comparison system:

$$
\begin{cases}\frac{d w_{2}(t)}{d t}=-D w_{2}(t), & t \neq n T, \\ w_{2}\left(t^{+}\right)=w_{2}(t)+\gamma F_{0}, & t=n T \\ w_{2}\left(0^{+}\right)=F\left(0^{+}\right), & \end{cases}
$$

then we have $F(t) \leq w_{2}(t)$ and $w_{2}(t) \rightarrow F^{*}(t)$. Then, for any $\varepsilon_{2}>0$, there exists $T^{\prime \prime}>0$ such that

$$
F(t) \leq F^{*}(t)-\varepsilon_{2}
$$

for $t>T^{\prime \prime}$. Thus, by (16) and (18), for $t>\max \left\{T^{\prime}, T^{\prime \prime}\right\}$, we have

$$
w_{1}^{*}(t)-\varepsilon_{2} \leq F(t) \leq F^{*}(t)-\varepsilon_{2} .
$$

Let $\varepsilon \rightarrow 0$, we have

$$
F^{*}(t)-\varepsilon_{2}<F(t)<F^{*}(t)+\varepsilon_{2}
$$

for $t$ large enough, thus we get $\lim _{t \rightarrow \infty} F(t)=F_{0}$. This completes the proof.

\subsection{Permanence}

In this section, we prove that system (2) is persistent for $R_{2}>1$. Firstly, we give the following lemma supporting our main conclusion. Denote

$$
R_{2}=\frac{h e^{-D \tau} S_{0}}{D+h_{2} \frac{\gamma F_{0}}{1-e^{-D T}}}
$$


Lemma 3.1 If $R_{2}>1$, then there exists a constant $m_{4}>0$ such that

$$
\liminf _{t \rightarrow \infty} x(t) \geq \min \left\{\frac{m_{4}}{2}, m_{4} e^{-\left(D+h_{2} \varrho_{2}\right) \tau}\right\}=m_{2} .
$$

Proof Let $X(t)=(S(t), x(t), F(t))$ be any positive solution of system (2) with initial condition (3). We rewrite the second equation of system (2) as follows:

$$
\frac{d x(t)}{d t}=\left(e^{-D \tau} h S(t)-D-h_{2} F(t)\right) x(t)-e^{-D \tau} h \int_{t-\tau}^{t} x(\sigma) S(\sigma) d \sigma .
$$

Define

$$
G(t)=x(t)+e^{-D \tau} h \int_{t-\tau}^{t} x(\sigma) S(\sigma) d \sigma .
$$

Calculating the derivative of $G(t)$ along the solution of (2) yields

$$
\frac{d G(t)}{d t}=\left(e^{-D \tau} h S(t)-D-h_{2} F(t)\right) x(t) .
$$

Let $m_{4}=\frac{\left(R_{2}-1\right) D}{\left(R_{2}+1\right) h_{1}}$. Since $R_{2}>1$, it is clear that $m_{4}>0$. For $m_{4}$, one can choose $\varepsilon_{1}, \varepsilon_{2}>0$ small enough such that

$$
\frac{e^{-D \tau} h \varrho_{1}}{D+h_{2} \varrho_{2}}>1
$$

where $\varrho_{1}=\frac{D S_{0}}{D+h_{1} m_{4}}-\varepsilon_{1}, \varrho_{2}=\frac{\gamma F_{0}}{1-e^{-D T}}+\varepsilon_{2}$. Then, for any positive constant $t_{0}$ and for all $t \geq t_{0}$, we claim that the inequality $x(t)<m_{4}$ cannot hold. Otherwise, there must exist a positive constant $t_{0}$ such that $x(t)<m_{4}$ for all $t \geq t_{0}$. The first equation of system (2) leads to

$$
\frac{d S(t)}{d t} \geq D S_{0}-\left(D+h_{1} m_{4}\right) S(t)
$$

By Lemma 2.3, there exists such $T_{1}>t_{0}+\tau$ for $t>T_{1}$ that

$$
S(t)>\frac{D S_{0}}{D+h_{1} m_{4}}-\varepsilon_{1}=\varrho_{1} .
$$

From (18), there exists such $T_{2}>t_{0}+\tau$ for $t \geq T_{2}$ that

$$
F(t)<F^{*}(t)+\varepsilon_{2}=\frac{\gamma F_{0} e^{-D(t-n T)}}{1-e^{-D T}}+\varepsilon_{2}<\frac{\gamma F_{0}}{1-e^{-D T}}+\varepsilon_{2}=\varrho_{2} .
$$

Thus by (21), (22) and (19), for $t>T_{3}=\max \left\{T_{1}, T_{2}\right\}$, one gets

$$
\frac{d G(t)}{d t} \geq\left(D+h_{2} \varrho\right)\left(\frac{e^{-D \tau} \varrho_{1}}{D+h_{2} \varrho_{2}}-1\right) x(t) .
$$

Let

$$
x_{l}=\min _{t \in\left[T_{1}, T_{1}+\tau\right]} x(t) .
$$


We can prove that $x(t) \geq x_{l}$ for all $t \geq T_{3}$. Otherwise, there exists a constant $T_{4} \geq 0$ such that $x(t) \geq x_{l}$ for $t \in\left[T_{3}, T_{3}+\tau+T_{4}\right], x\left(T_{3}+\tau+T_{4}\right)=x_{l}$ and $\dot{x}\left(T_{3}+\tau+T_{4}\right) \leq 0$. Then from the second equation of (2), (20) and (23), we have

$$
\begin{aligned}
\dot{x}\left(T_{3}+\tau+T_{4}\right) & >\left(e^{-D \tau} h \varrho_{1}-D-h_{2} \varrho_{2}\right) x_{l} \\
& =\left(D+h_{2} \varrho_{2}\right)\left(\frac{e^{-D \tau} h \varrho_{1}}{D+h_{2} \varrho_{2}}-1\right) x_{l} \\
& >0,
\end{aligned}
$$

which is a contradiction. Hence $x(t) \geq x_{l}>0$ for all $t \geq T_{3}$. Inequality (23) implies

$$
\frac{d G(t)}{d t} \geq\left(D+h_{2} \varrho\right)\left(\frac{e^{-D \tau} \varrho_{1}}{D+h_{2} \varrho_{2}}-1\right) x_{l}>0
$$

which implies $G(t) \rightarrow+\infty$ as $t \rightarrow+\infty$. This is a contradiction to $G(t) \leq M\left(1+M \tau e^{-D M}\right)$ for $t$ large enough. Therefore, for any positive constant $t_{0}$, the inequality $x(t)<m_{4}$ cannot hold for all $t \geq t_{0}$.

Step II: From Step I, we only need to consider:

(i) $x(t)>m_{4}$ for all $t$ large enough;

(ii) $x(t)$ oscillates about $m_{4}$ for all large $t$.

However, case (i) is obviously the result of this theorem, so we only need to consider case (ii), in which we shall show that $x(t)>m_{2}$ for all large $t$, where

$$
m_{2}=\min \left\{\frac{m_{4}}{2}, m_{4} e^{-\left(D+h_{2} \varrho_{2}\right) \tau}\right\}
$$

First, we notice that there would be two positive arbitrarily big constants $\bar{t}, \varphi$ such that $x(t)<m_{4}$ for $\bar{t}<t<\bar{t}+\varphi$ and $x(\bar{t})=x(\bar{t}+\varphi)=m_{4}$. Second, there exists a constant $0<T_{5}<\tau$ such that $x(t)>\frac{m_{4}}{2}$ for all $\bar{t} \leq t \leq \bar{t}+T_{5}$. Because $x(t)$ is not affected by impulses and, moreover, $x(t)$ is bound and continuous, then we conclude that $T_{5}$ is independent of the choice of $\bar{t}$. Next, according to the position of $\varphi, T_{5}, \tau$, there will be three cases we should discuss.

Case ii(a): $\varphi \leq T_{5}$, obviously our aim is obtained.

Case ii(b): $T_{5}<\varphi \leq \tau$. By (22), the second equation of (2) implies

$$
\dot{x}(t) \geq-\left(D+h_{2} \varrho_{2}\right) x(t)
$$

for $\bar{t}<t \leq \bar{t}+\varphi<\bar{t}+\tau$. Then we have

$$
x(t) \geq x(\bar{t}) e^{-\left(D+h_{2} \varrho_{2}\right) \tau}
$$

for $\bar{t}<t \leq \bar{t}+\varphi \leq \bar{t}+\tau$, notice $x(\bar{t})=m_{4}$, one can get

$$
x(t) \geq m_{4} e^{-\left(D+h_{2} \varrho_{2}\right) \tau}
$$

for $\bar{t}<t \leq \bar{t}+\varphi \leq \bar{t}+\tau$. Thus we have $x(t) \geq m_{2}$ for $\bar{t}<t \leq \bar{t}+\varphi$. 
Case ii (c): $\varphi \geq \tau$. We have proved $x(t) \geq m_{2}$ for $\bar{t}<t \leq \bar{t}+\tau$. For $\bar{t}+\tau \leq t \leq \bar{t}+\varphi$, we can analyze and prove $x(t) \geq m_{2}$ as the proof for the above claim. Because of the arbitrariness of interval $[\bar{t}, \bar{t}+\varphi]$ and because $\bar{t}$ is an arbitrarily big constant, we have that $x(t) \geq m_{2}$ holds for $t$ large enough. Finally, notice that the choice of $m_{2}$ is independent of the positive solution of (2), which satisfies that $x(t) \geq m_{2}$ for $t$ large enough. This completes the proof of Lemma 2.4.

Theorem 3.3 For $R_{2}>1$, then system (2) will be permanent.

Proof Let $X(t)=(S(t), x(t), F(t))$ be any positive solution of system (2) with initial condition (3). From the first equation of system (2), we get

$$
\frac{d S(t)}{d t} \geq D S_{0}-\left(D+h_{1} M\right) S(t)
$$

then we have

$$
\liminf _{t \rightarrow \infty} S(t) \geq \frac{D S_{0}}{D+h_{1} M}
$$

Thus there exists a constant $\varepsilon$ small enough such that

$$
S(t)>\frac{D S_{0}}{D+h_{1} M}-\varepsilon=m_{1}>0
$$

for $t$ large enough. And from (13) we have

$$
F(t) \geq z(t)>z^{*}(t)-\varepsilon>\frac{\gamma F_{0} e^{-\left(D+h_{3} M\right) T}}{1-e^{-\left(D+h_{3} M\right) T}}=m_{3}>0
$$

for $t$ large enough.

Set

$$
D=\left\{(S, x, F) \in R_{+}^{3} \mid m_{1} \leq S \leq M_{1}, m_{2} \leq x \leq M_{2}, m_{3} \leq F \leq M_{3}\right\}
$$

Thus $D$ is a bounded compact region and every solution of system (2) will eventually enter and remain in region $D$, then system (2) is permanent. The proof of Theorem 3.3 is completed.

\section{Control strategy of continuous microbial culture and harvest}

In Section 3, we obtain the threshold values $R_{1}$ and $R_{2}$ associated with microbial extinction and existence. Next, we discuss the control strategy of continuous microbial culture and harvest by analyzing the key parameters of the threshold.

Denote

$$
\begin{aligned}
T^{*} & =\frac{1}{D+h_{3} M_{2}} \ln \frac{h e^{-D \tau} S_{0}+h_{2} \gamma F_{0}-D}{h e^{-D \tau} S_{0}-D}, \\
F_{0}^{*} & =\frac{h e^{-D \tau} S_{0}-D}{h_{2} \frac{\gamma e^{-\left(D+h_{3} M_{2}\right) T}}{1-e^{-\left(D+h_{3} M_{2}\right) T}}},
\end{aligned}
$$




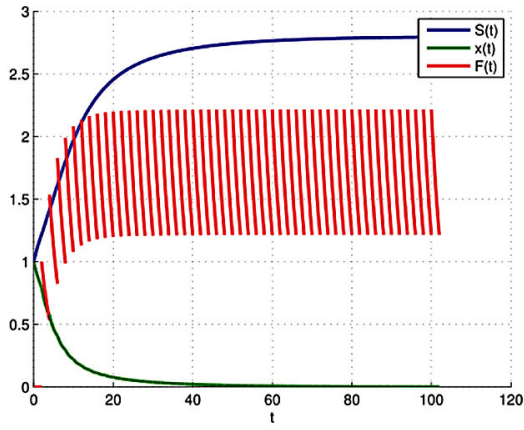

(a)

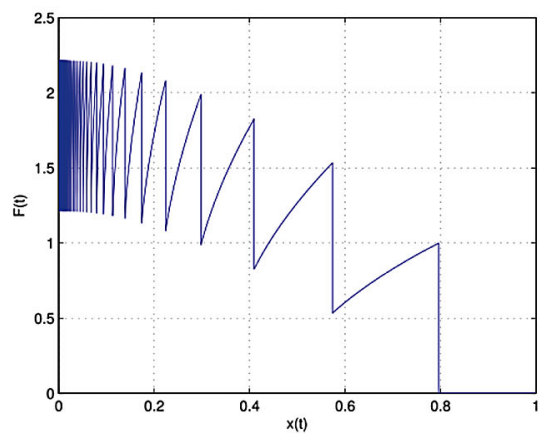

(c)

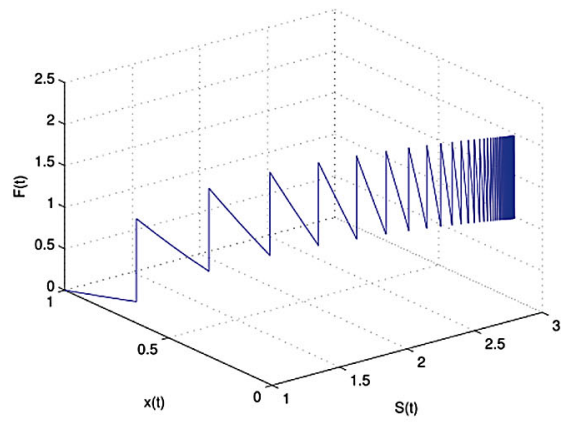

(b)

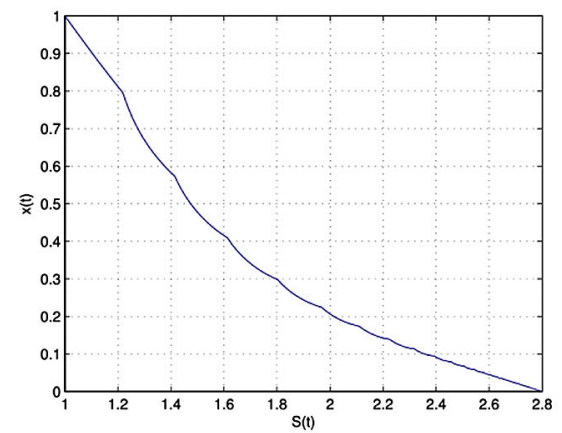

(d)

Figure 3 Dynamical behavior of solutions of system (26) with $\boldsymbol{R}_{\mathbf{1}}=\mathbf{0 . 9 9 1 0}<\mathbf{1}$. (a) Time series of $S(t), x(t)$, $F(t)$. (b) Three-dimensional phase diagrams of $S(t), x(t), F(t)$. (c) Phase diagrams of $x(t), F(t)$. (d) Phase diagrams of $x(t), S(t)$.

$$
\begin{aligned}
\tau^{*} & =\frac{1}{D} \ln \frac{h S_{0}}{D+h_{2} \frac{\gamma F_{0} e^{-\left(D+h_{3} M_{2}\right) T}}{1-e^{-\left(D+h_{3} M_{2}\right) T}},} \\
T_{*} & =\frac{1}{D} \ln \frac{h e^{-D \tau} S_{0}+h_{2} \gamma F_{0}-D}{h e^{-D \tau} S_{0}-D}, \\
F_{0 *} & =\frac{\left(h e^{-D \tau} S_{0}-D\right)\left(1-e^{-D T}\right)}{h_{2} \gamma}, \\
\tau_{*} & =\frac{1}{D} \ln \frac{h S_{0}}{D+h_{2} \frac{\gamma F_{0}}{1-e^{-D T}}} .
\end{aligned}
$$

According to Theorem 3.2, we have that if $T<T^{*}$ or $F_{0}>F_{0}^{*}$ or $\tau>\tau^{*}$, the microbeextinction periodic solution $\left(S_{0}, 0, F^{*}(t)\right)$ is globally attractive. That means the microbial continuous cultivation and harvest have failed. And from Theorem 3.3, we know that if $T>T_{*}$ or $F_{0}<F_{0 *}$ or $\tau<\tau_{*}$, then system (2) is permanent. That means we can achieve the process of microbial cultivation by increasing the time interval, or reducing the input amount of flocculant, or shortening the growth delay.

\section{Discussion and numerical simulations}

In this paper, to achieve the continuous microbial culture and harvest, we improve the classic chemostat model and propose a new chemostat model with time delay and peri- 


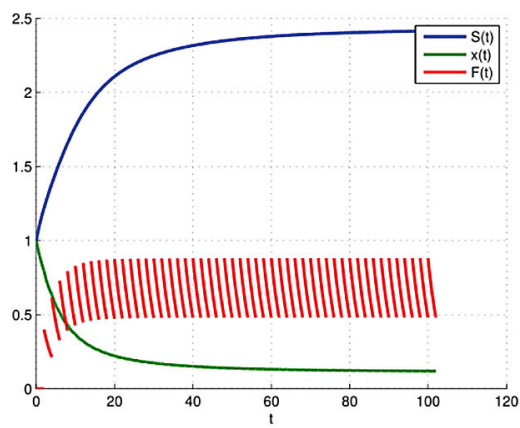

(a)

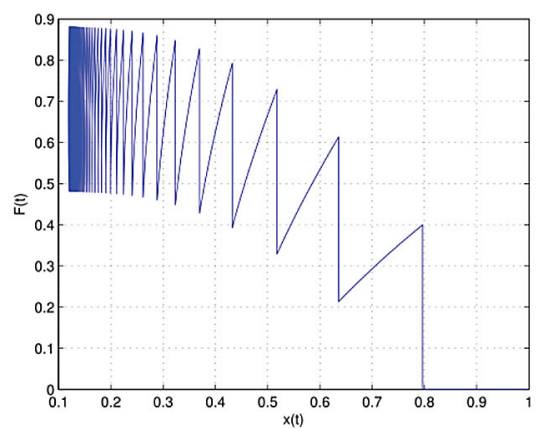

(c)

Figure 4 Dynamical behavior of solutions of system (26) with $\boldsymbol{R}_{\mathbf{2}}=\mathbf{1 . 0 6 4 7}>\mathbf{1}$. (a) Time series of $S(t), x(t)$ $F(t)$. (b) Three-dimensional phase diagrams of $S(t), x(t), F(t)$. (c) Phase diagrams of $x(t), F(t)$. (d) Phase diagrams of $x(t), S(t)$

(b)

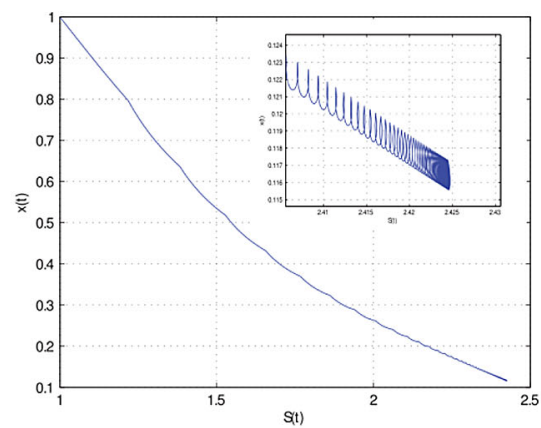

(d)

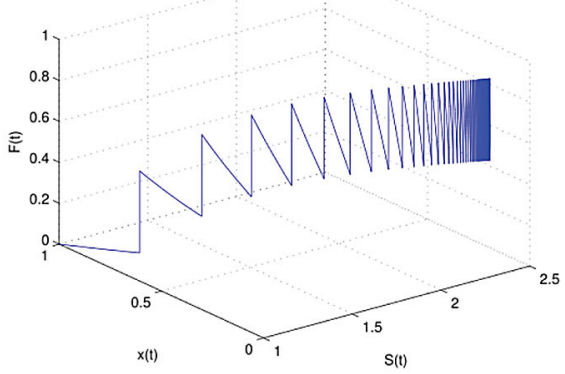

odical flocculant input. Our main aim is to investigate the control strategy of continuous microbial culture and harvest. By using the theory of impulsive delayed differential equations, global properties of the system are discussed. We prove that if $R_{1}<1$, then the microbe will be eventually extinct, and if $R_{2}>1$, the microbe species is permanent. Based on the threshold values associated with microbial extinction and existence, we consider the control strategy. Results show that we can culture microbia continuously and harvest microbia many times by adjusting the time interval $(T)$ or the input amount of flocculant $\left(\gamma F_{0}\right)$, or the time delay $(\tau)$.

Next we will verify the effectiveness of control strategy by an example and some numerical simulations. Let $D=0.3, S_{0}=2.8, h_{1}=0.4, h=0.3, h_{2}=0.15, h_{3}=0.02, \gamma=1$, and let the initial value be $(1,1,0)$. We get the following system:

$$
\left\{\begin{array}{l}
\frac{d S(t)}{d t}=0.3(2.8-S(t))-0.4 x(t) S(t), \\
\frac{d x(t)}{d t}=0.3 e^{-0.3 \tau} x(t-\tau) S(t-\tau)-0.3 x(t)-0.15 x(t) F(t), \\
\frac{d F(t)}{d t}=-0.3 F(t)-0.02 x(t) F(t), \\
S\left(t^{+}\right)=S(t), \\
x\left(t^{+}\right)=x(t), \\
F\left(t^{+}\right)=F(t)+\gamma F_{0},
\end{array}\right\} t=n T .
$$




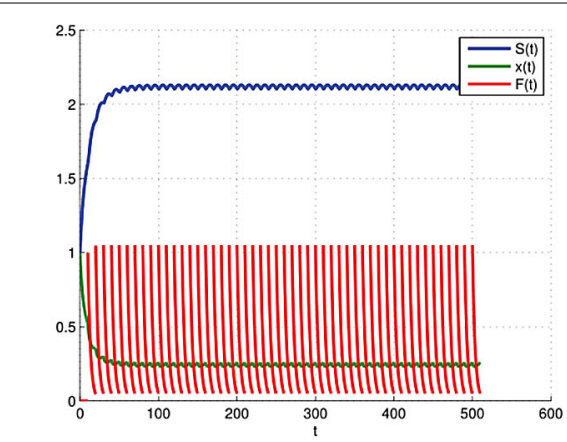

(a)

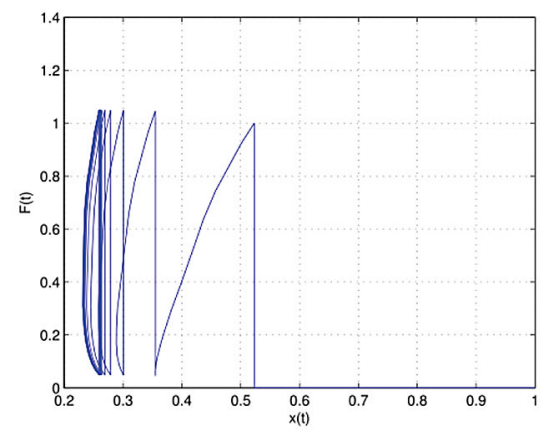

(c)

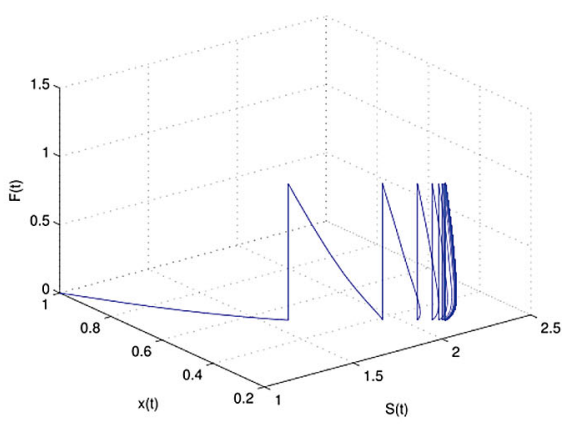

(b)

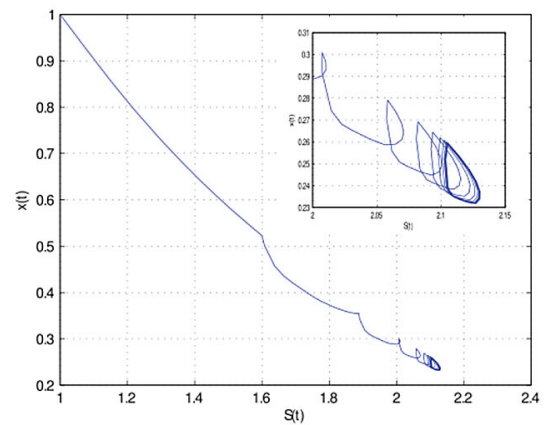

(d)

Figure 5 Dynamical behavior of solutions of system (26) with $\boldsymbol{R}_{\mathbf{2}}=\mathbf{1 . 0 0 6 9}>\mathbf{1}$. (a) Time series of $S(t), x(t)$, $F(t)$. (b) Three-dimensional phase diagrams of $S(t), x(t), F(t)$. (c) Phase diagrams of $x(t), F(t)$. (d) Phase diagrams of $x(t), S(t)$.

To investigate the effects of key parameters on the system, we assume $\tau=2, T=2$, $F_{0}=1$. By simple calculation, we have $R_{1}=0.9910<1$. Figure 3 shows that the microbeeradication periodic solution $\left(S_{0}, 0, F^{*}(t)\right)$ is globally attractive. That means microbial continuous cultivation and harvest have failed because the microbe will be eventually extinct. To achieve microbial continuous cultivation and harvest, we can take three kinds of control strategies.

(i) We can reduce the input of flocculant $F_{0}$ (from 1 to 0.4 ). By calculating, we have $R_{2}=1.0647>1$. According to Theorem 3.3, system (26) is permanent. A lower amount of flocculant can increase population microbia in the medium so that microbe can be cultured continuously in the chemostat system. Figure 4 shows that system (26) is permanent.

(ii) We can increase the time travail $T$ (from 2 to 10). Longer time travail can decrease input mount of flocculant indirectly and increase population microbia in the medium, which makes microbia cultured and harvested continuously. By calculating, we have $R_{2}=1.0069>1$. According to Theorem 3.3, system (26) is permanent (see Figure 5). Figure 5 also shows that system (26) has an asymptotically stable periodic solution.

(iii) We can decrease time delay $\tau$ (from 2 to 0.5 ) by some biotechnology and biological engineering. Reduction of growth time delay makes the microbial growth cycle shorter, which is beneficial for microbial continuous cultivation. Figure 6 shows that system (26) is permanent. Moreover, system (26) has an asymptotically stable 


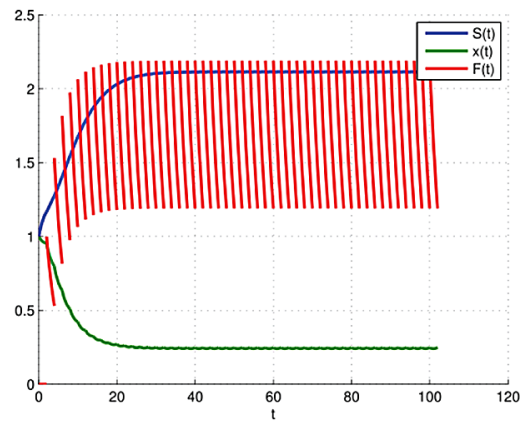

(a)

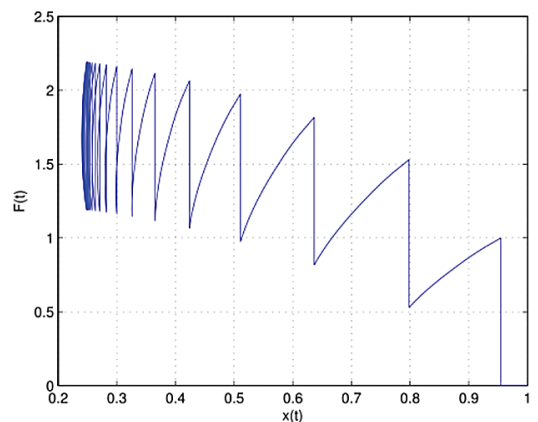

(c)

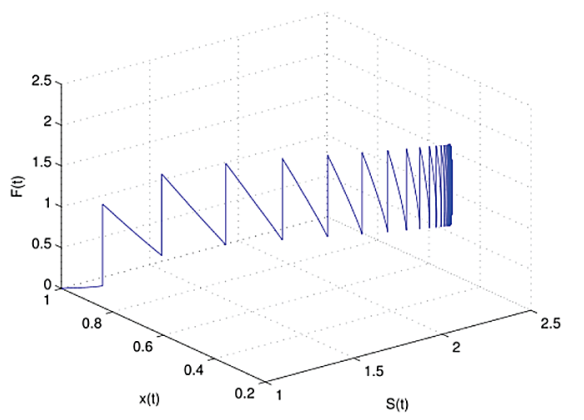

(b)

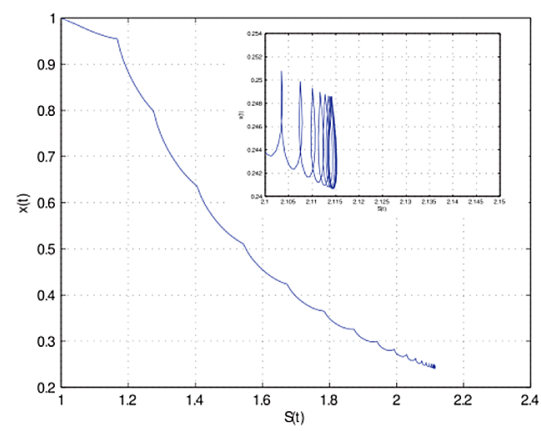

(d)

Figure 6 Dynamical behavior of solutions of system (26) with $R_{\mathbf{2}}=1.1432>1$. (a) Time series of $S(t), x(t)$, $F(t)$. (b) Three-dimensional phase diagrams of $S(t), x(t), F(t)$. (c) Phase diagrams of $x(t), F(t)$. (d) Phase diagrams of $x(t), S(t)$

Table 1 Values of parameters, threshold and state of the system

\begin{tabular}{lllllllrlllll}
\hline $\boldsymbol{\tau}$ & $\boldsymbol{D}$ & $\boldsymbol{S}_{\mathbf{0}}$ & $\boldsymbol{h}_{\mathbf{1}}$ & $\boldsymbol{h}$ & $\boldsymbol{h}_{\mathbf{2}}$ & $\boldsymbol{h}_{\mathbf{3}}$ & $\boldsymbol{T}$ & $\boldsymbol{\gamma}$ & $\boldsymbol{F}_{\mathbf{0}}$ & $\boldsymbol{R}_{\boldsymbol{i}}(\boldsymbol{i}=\mathbf{1 , 2})$ & Microbe & Figure \\
\hline 2 & 0.3 & 2.8 & 0.4 & 0.3 & 0.15 & 0.02 & 2 & 1 & 1 & $R_{1}=0.9910<1$ & Eradication & Figure 3 \\
2 & 0.3 & 2.8 & 0.4 & 0.3 & 0.15 & 0.02 & 2 & 1 & 0.4 & $R_{2}=1.0647>1$ & Permanence & Figure 4 \\
2 & 0.3 & 2.8 & 0.4 & 0.3 & 0.15 & 0.02 & 10 & 1 & 1 & $R_{2}=1.0069>1$ & Permanence & Figure 5 \\
0.5 & 0.3 & 2.8 & 0.4 & 0.3 & 0.15 & 0.02 & 2 & 1 & 1 & $R_{2}=1.1432>1$ & Permanence & Figure 6 \\
\hline
\end{tabular}

periodic solution (where $R_{2}=1.1432>1$ ). Detailed parameter values, thresholds and states of system (26), please see Table 1.

\section{Competing interests}

The authors declare that they have no competing interests.

Authors' contributions

TZ and WM designed the study and carried out the analysis. TZ, WM and XM contributed to writing the paper. TZ performed numerical simulations. All authors read and approved the final manuscript.

\section{Author details}

1 School of Mathematics and Physics, University of Science and Technology Beijing, 30 Xue Yuan Road, Beijing, 100083

China. ${ }^{2}$ College of Mathematics and Systems Science, Shandong University of Science and Technology, 579

Qianwangang Road, Qingdao, 266510, China. ${ }^{3}$ State Key Laboratory of Mining Disaster Prevention and Control

Co-founded by Shandong Province and the Ministry of Science and Technology, Shandong University of Science and

Technology, 579 Qianwangang Road, Qingdao, 266510, China.

\section{Acknowledgements}

The first author and the third author were supported by the National Natural Science Foundation of China (No. 11371230),

Shandong Provincial Natural Science Foundation, China (No. ZR2015AQ001), the Project for Higher Educational Science 
and Technology Program of Shandong Province of China (No. J13LI05), SDUST Research Fund (2014TDJH102), and Joint Innovative Center for Safe and Effective Mining Technology and Equipment of Coal Resources, Shandong Province of China. The second author was supported by the National Natural Science Foundation of China (No. 11471034).

\section{Publisher's Note}

Springer Nature remains neutral with regard to jurisdictional claims in published maps and institutional affiliations.

Received: 26 December 2016 Accepted: 29 March 2017 Published online: 20 April 2017

\section{References}

1. Novick, A, Szilard, L: Description of the chemostat. Science 112(2920), 715-716 (1950)

2. James, TW: Continuous culture of microorganisms. Annu. Rev. Microbiol. 15(1), 27-46 (1961)

3. Monod, J: Recherches sur la croissance des cultures bacteriennes. Hermann, Paris (1942)

4. Butler, GJ, Wolkowicz, GSK: A mathematical model of the chemostat with a general class of functions describing nutrient uptake. SIAM J. Appl. Math. 45(1), 138-151 (1985)

5. Hsu, SB, Hubbell, S, Waltman, P: A mathematical theory for single-nutrient competition in continuous cultures of micro-organisms. SIAM J. Appl. Math. 32(2), 366-383 (1977)

6. Levins, R: Coexistence in a variable environment. Am. Nat. 114(6), 765-783 (1979)

7. Wolkowicz, GSK, Lu, Z: Global dynamics of a mathematical model of competition in the chemostat: general response functions and differential death rates. SIAM J. Appl. Math. 52(1), 222-233 (1992)

8. Smith, HL, Waltman, P: The Theory of the Chemostat: Dynamics of Microbial Competition. Cambridge University Press, Cambridge (1995)

9. Sun, S, Chen, L: Dynamic behaviors of Monod type chemostat model with impulsive perturbation on the nutrient concentration. J. Math. Chem. 42(4), 837-847 (2007)

10. Luo, J, Yuan, S, Zhang, W: Competition between two microorganisms in the chemostat with general variable yields and general growth rates. Int. J. Biomath. 1(4), 463-474 (2008)

11. Zhao, Z, Chen, L, Song, $X$ : Extinction and permanence of chemostat model with pulsed input in a polluted environment. Commun. Nonlinear Sci. Numer. Simul. 14(4), 1737-1745 (2009)

12. Teng, Z, Gao, R, Rehim, M, Wang, K: Global behaviors of Monod type chemostat model with nutrient recycling and impulsive input. J. Math. Chem. 47(1), 276-294 (2009)

13. Meng, X, Wang, L, Zhang, T: Global dynamics analysis of a nonlinear impulsive stochastic chemostat system in a polluted environment. J. Appl. Anal. Comput. 6(3), 865-875 (2016)

14. Li, Z, Wang, T, Chen, L: Periodic solution of a chemostat model with Beddington-DeAnglis uptake function and impulsive state feedback control. J. Theor. Biol. 261(1), 23-32 (2009)

15. Sun, K, Tian, Y, Chen, L, Kasperski, A: Nonlinear modelling of a synchronized chemostat with impulsive state feedback control. Math. Comput. Model. 52(1), 227-240 (2010)

16. Zhang, T, Zhang, T, Meng, X: Stability analysis of a chemostat model with maintenance energy. Appl. Math. Lett. 68, $1-7(2017)$

17. Grover, J: Resource Competition. Chapmam \& Hall, London (1997)

18. Hansen, SR, Hubbell, SP: Single-nutrient microbial competition: qualitative agreement between experimental and theoretically forecast outcomes. Science 207(4438), 1491-1493 (1980)

19. Tilman, D: Resource Competition and Community Structure. Princeton University Press, Princeton (1982)

20. Li, Z, Chen, L, Liu, Z: Periodic solution of a chemostat model with variable yield and impulsive state feedback control. Appl. Math. Model. 36(3), 1255-1266 (2012)

21. Morales, J, de la Noue, J, Picard, G: Harvesting marine microalgae species by chitosan flocculation. Aquac. Eng. 4(4), 257-270 (1985)

22. Yan, H, Ma, C, Sun, X, Chen, J, Wang, D: Study on flocculation of Rhodopeudomonas palustris by aluminum flocculants. Chem. Bioeng. 25(6), 53-55 (2008)

23. Vandamme, D, Foubert, I, Muylaert, K: Flocculation as a low-cost method for harvesting microalgae for bulk biomass production. Trends Biotechnol. 31(4), 233-239 (2013)

24. Choi, J-H, Shin, WS, Lee, S-H, Joo, D-J, Lee, J-D, Choi, SJ, Park, LS: Application of synthetic polyamine flocculants for dye wastewater treatment. Sep. Sci. Technol. 36(13), 2945-2958 (2001)

25. Zheng, H, Gao, Z, Yin, J, Tang, X, Ji, X, Huang, H: Harvesting of microalgae by flocculation with poly ( $\gamma$-glutamic acid). Bioresour. Technol. 112, 212-220 (2012)

26. Salim, S, Bosma, R, Vermuë, MH, Wijffels, RH: Harvesting of microalgae by bio-flocculation. J. Appl. Phycol. 23(5), 849-855 (2011)

27. Lee, CS, Robinson, J, Chong, MF: A review on application of flocculants in wastewater treatment. Process Saf. Environ. Prot. 92(6), 489-508 (2014)

28. Rahul, R, Jha, U, Sen, G, Mishra, S: Carboxymethyl inulin: a novel flocculant for wastewater treatment. Int. J. Biol. Macromol. 63, 1-7 (2014)

29. Daud, Z, Nasir, N, Awang, H, et al.: Treatment of biodiesel wastewater by coagulation and flocculation using polyaluminum chloride. Aust. J. Basic Appl. Sci. 7(8), 258-262 (2013)

30. Sen, G, Rani, GU, Mishra, S: Microwave assisted synthesis of poly(2-hydroxyethylmethacrylate) grafted agar (Ag-g-P(HEMA)) and its application as a flocculant for wastewater treatment. Front. Chem. Sci. Eng. 7(3), 312-321 (2013)

31. Deng, S, Bai, R, Hu, X, Luo, Q: Characteristics of a bioflocculant produced by Bacillus mucilaginosus and its use in starch wastewater treatment. Appl. Microbiol. Biotechnol. 60(5), 588-593 (2003)

32. Wang, J-P, Chen, Y-Z, Yuan, S-J, Sheng, G-P, Yu, H-Q: Synthesis and characterization of a novel cationic chitosan-based flocculant with a high water-solubility for pulp mill wastewater treatment. Water Res. 43(20), 5267-5275 (2009)

33. Tai, X, Ma, W, Guo, S, Yan, H: A class of dynamic delayed model describing flocculation of microorganism and its theoretical analysis. Math. Pract. Theory 13(45), 198-209 (2015) 
34. Finn, RK, Wilson, RE: Fermentation process control, population dynamics of a continuous propagator for microorganisms. J. Agric. Food Chem. 2(2), 66-69 (1954)

35. Bush, AW, Cool, AE: The effect of time delay and growth rate inhibition in the bacterial treatment of wastewater J. Theor. Biol. 63(2), 385-395 (1976)

36. Cunningham, A, Nisbet, RM: Time lag and co-operativity in the transient growth dynamics of microalgae. J. Theor. Biol. 84(2), 189-203 (1980)

37. Ellermeyer, SF: Competition in the chemostat: global asymptotic behavior of a model with delayed response in growth. SIAM J. Appl. Math. 54(2), 456-465 (1994)

38. Freedman, $\mathrm{HI}, \mathrm{So}, \mathrm{JW}-\mathrm{H}$, Waltman, P: Coexistence in a model of competition in the chemostat incorporating discrete delays. SIAM J. Appl. Math. 49(3), 859-870 (1989)

39. Gopalsamy, K, Aggarwala, BD: Limit cycles in two spacies competition with time delays. J. Aust. Math. Soc. Ser. B, Appl. Math 22(2), 148-160 (1980)

40. Macdonald, N: Time delay in simple chemostat models. Biotechnol. Bioeng. 18(6), 805-812 (1976)

41. Ruan, S: The effect of delays on stability and persistence in plankton models. Nonlinear Anal. 24(4), 575-585 (1995)

42. Jiao, J, Yang, X, Chen, L, Cai, S: Effect of delayed response in growth on the dynamics of a chemostat model with impulsive input. Chaos Solitons Fractals 42(4), 2280-2287 (2009)

43. Meng, X, Gao, Q, Li, Z: The effects of delayed growth response on the dynamic behaviors of the Monod type chemostat model with impulsive input nutrient concentration. Nonlinear Anal., Real World Appl. 11(5), 4476-4486 (2010)

44. Dong, $\mathrm{Q}, \mathrm{Ma}, \mathrm{W}$, Sun, M: The asymptotic behavior of a chemostat model with Crowley-Martin type functional response and time delays. J. Math. Chem. 51(5), 1231-1248 (2013)

45. Cao, J, Bao, J, Wang, P: Global analysis of a delayed Monod type chemostat model with impulsive input on two substrates. Adv. Differ. Equ. 2015, Article ID 310 (2015)

46. Zhang, T, Meng, X, Zhang, T, Song, Y: Global dynamics for a new high-dimensional SIR model with distributed delay. Appl. Math. Comput. 218(24), 11806-11819 (2012)

47. Zhang, T, Meng, X, Song, Y, Zhang, T: A stage-structured predator-prey SI model with disease in the prey and impulsive effects. Math. Model. Anal. 18(4), 505-528 (2013)

48. Braverman, E, Mamdani, R: Continuous versus pulse harvesting for population models in constant and variable environment. J. Math. Biol. 57(3), 413-434 (2008)

49. Tian, Y, Sun, K, Chen, L, Kasperski, A: Studies on the dynamics of a continuous bioprocess with impulsive state feedback control. Chem. Eng. J. 157(2), 558-567 (2010)

50. Guo, H, Chen, L: Periodic solution of a chemostat model with Monod growth rate and impulsive state feedback control. J. Theor. Biol. 260(4), 502-509 (2009)

51. Cheng, H, Zhang, T: A new predator-prey model with a profitless delay of digestion and impulsive perturbation on the prey. Appl. Math. Comput. 217(22), 9198-9208 (2011)

52. Lakshmikantham, V, Bainov, DD, Simeonov, PS: Theory of Impulsive Differential Equations. World Scientific, Singapore (1989)

53. Bainov DD, Simeonov PS: Systems with Impulse Effect: Stability, Theory, and Applications. E. Horwood Limited, Chichester (1989)

54. Kuang, Y: Delay Differential Equations: With Applications in Population Dynamics. Academic Press, New York (1993)

\section{Submit your manuscript to a SpringerOpen ${ }^{\circ}$ journal and benefit from:}

- Convenient online submission

Rigorous peer review

- Immediate publication on acceptance

- Open access: articles freely available online

- High visibility within the field

- Retaining the copyright to your article 